\title{
IMPACTO EM LONGO PRAZO DA INFECÇÃO DO VÍRUS DA HEPATITE C EM PACIENTES SUBMETIDOS A TRANSPLANTE RENAL
}

\author{
Long-Term Impact In Hepatitis C Virus Infection In Post transplantation
}

\begin{abstract}
Euler Pace Lasmar, Marcus Faria Lasmar, Leonardo Faria Lasmar, Adalberto Fernandes Nogueira, Marina Ribeiro de Oliveira Santos, Luiz Flávio Couto Giordano, Heloisa Reniers Vianna, Juliene Borges Fujji
\end{abstract}

\begin{abstract}
RESUMO
Objetivo: Avaliar o impacto da infecção crônica do vírus da hepatite C (HCV) na evolução do transplante (Tx) renal, estudando-se as complicações e a sobrevida do paciente e do enxerto. Métodos: Estudo retrospectivo observacional analisando 40 pacientes HCV positivos e 40 pacientes HCV negativos transplantados no mesmo período. Resultado: O tempo médio de transplante foi de 12,3 \pm 4,5 anos em pacientes com infecção por HCV e de 12,5 \pm 2,9 anos em pacientes sem infecção por HCV (p=0,49). Não houve diferença estatística significativa com relação à idade e sexo de receptores, idade e tipo de doadores. A função renal atual dos pacientes HCV positivos foi de 47,3 $\pm 24,9 \mathrm{ml} / \mathrm{min}$ e nos HCV negativos, 54,9 $\pm 27,2 \mathrm{ml} / \mathrm{min}(\mathrm{p}=0,48)$. A sobrevida do enxerto e dos pacientes foi semelhante em ambos os grupos. A principal causa de óbito nos dois grupos foi infecção bacteriana (10\% nos pacientes HCV positivos e 12,5\% nos HCV negativos; $\mathrm{p}=0,26)$. As principais complicações observadas nos dois grupos: rejeição aguda, infecção bacteriana e incidência de diabetes mellitus foi semelhante. Alterações nas enzimas hepáticas e cirrose ocorreram apenas em três pacientes com infecção por HCV. Conclusão: A infecção por vírus C não teve impacto na sobrevida do enxerto e dos pacientes, bem como em suas complicações.
\end{abstract}

Descritores: HCV, Transplante Renal, Falência Renal Crônica

Instituição:

Unidade de Transplante Renal do Hospital Universitário São José / Hospital Mater Dei Belo Horizonte / MG

\section{Correspondência:}

Euler Pace Lasmar

Rua Newtow, 89 - Belo Horizonte / MG - CEP: 30360-200 - Brasil

Tel.: (31) 32978848

E-mail: lasmar@superig.com.br

Recebido em: 16.06 .2008

Aceito em: 02.07.2008

\section{INTRODUÇÃO}

A Infecção por vírus da hepatite C (HCV) constitui a principal causa de doença hepática em pacientes portadores de insuficiência renal crônica em programa de hemodiálise e em transplantados renais. A prevalência de $\mathrm{HCV}$ varia de acordo com centros e países, sendo de $3 \%$ a $80 \%$ nos pacientes em diálise e 10 a $41 \%$ em receptores de transplante renal. ${ }^{1,2}$

Pacientes HCV positivos na lista de espera para Tx apresentam maior risco de mortalidade do que aqueles submetidos a Tx, risco que aumenta com o tempo de espera. ${ }^{3,4}$

Apesar da melhora no resultado do Tx renal, várias publicações têm mostrado que a insuficiência hepática secundária à infecção por HCV representa uma das principais causas de morbidade e mortalidade em pacientes imunossuprimidos. ${ }^{5}$

É controverso na literatura, o impacto do HCV na sobrevida do paciente e do enxerto no curto e longo prazos. ${ }^{6,7,8,9,10,11}$

O objetivo deste trabalho foi observar o impacto da infecção crônica do vírus HCV na evolução do Tx renal, considerando-se a sobrevida do paciente e do enxerto, bem como eventuais complicações.

\section{MATERIAL E MÉTODO}

Foi analisada a evolução clínica de 40 pacientes HCV positivos e 40 pacientes $\mathrm{HCV}$ negativos, selecionados retrospectivamente em 
um mesmo período de realização dos transplantes até o presente momento. Os pacientes HCV positivos foram submetidos a biópsia hepática prévia ao Tx, sendo que nenhum apresentava histologia, hepatite crônica ativa ou cirrose.

Foram analisadas as seguintes variáveis: idade, sexo e cor dos receptores, tipo do doador, tipagem HLA, tempo do Tx, função renal atual, sobrevida do enxerto e do paciente, tempo e causa da perda do enxerto e dos pacientes, imunossupressão utilizada e complicações observadas.

O teste do anticorpo anti-HCV foi realizado prévia e seriadamente após o Tx, através do método Elisa de primeira geração no período de 1990 a 1992, segunda geração durante 1992 a 1997 e terceira geração em seguida. A função renal foi avaliada pela depuração de creatinina através da fórmula de Cochroft Gault.

A fenotipagem do vírus C não foi feita, por se tratar de estudo retrospectivo.

As causas da insuficiência renal foram indeterminadas em 37 pacientes $(46,2 \%)$; glomerulonefrite crônica em 26 pacientes (32,5\%); doença policística renal, cinco pacientes $(12,5 \%)$; nefroesclerose hipertensiva, cinco pacientes (12,5\%); nefrite intersticial crônica, quatro pacientes $(1,0 \%)$; nefropatia diabética, dois pacientes $(0,5 \%)$ e doença de Alport, um paciente $(0,25 \%)$.

\section{ANÁLISE ESTATÍSTICA}

Para comparação entre os dois grupos em relação às variáveis quantitativas, utilizou-se o teste não paramétrico Mann-Whitney. Quando a comparação envolveu variável qualitativa foi utilizado o teste qui-quadrado ou teste exato de Fisher, sendo o nível de significância de $5 \%, \mathrm{p}=0,05$.

\section{RESULTADO}

O tempo médio de Tx foi de 12,3 \pm 4,5 anos nos pacientes $\mathrm{HCV}$ positivos e de 12,5 $\pm 2,9$ anos nos HCV negativos ( $p=0,49)$. A tabela 1 mostra as características demográficas de ambos os grupos. Não houve diferença estatística significativa com relação à idade e sexo do receptor, bem como da idade e tipo do doador, sendo que a incidência de HLA haploidêntico foi maior nos pacientes HCV negativos $(\mathrm{p}=0,05)$. A função renal atual nos HCV positivos foi 47,3 $\pm 24,9 \mathrm{ml} / \mathrm{min}$ e nos HCV negativos $54,9 \pm 27,2 \mathrm{ml} / \mathrm{min}(\mathrm{p}=0,48)$ e a sobrevida do enxerto foi semelhante em ambos os grupos, sendo de $30 \%$ nos pacientes HCV positivos e $40 \%$ nos pacientes HCV negativos $(\mathrm{p}=0,48)$ (tabela 1$)$. O tempo em meses até a perda do enxerto foi de 36,1 \pm 3,1 nos pacientes HCV positivos e 47,7 \pm 40,6 nos HCV negativos ( $p=0,67$ ), sendo que a principal causa foi óbito, idêntico em ambos os grupos (13 pacientes, 32,5\%), seguida da nefropatia crônica do enxerto (NCE), que teve maior incidência nos pacientes HCV negativos (30,0\%) do que nos HCV positivos (15,5\%); $\mathrm{p}=0,03$ (tabela 2). A sobrevida dos pacientes foi de $65 \%$ nos HCV positivos e $67,5 \%$ nos HCV negativos ( $p=0,22)$, sendo o óbito mais precoce nos pacientes HCV positivos $(18,0 \pm 30,2$ meses x 49,8 \pm 58,7 meses, $p=0,03$ ). A principal causa de óbito foi infecção bacteriana, semelhante em ambos os grupos, sendo 10,0\% nos HCV negativos e 12,5\% nos HCV positivos $(\mathrm{p}=0,63)$ (tabela 3). Não houve diferença significativa na imunossupressão entre grupos $(p=0,87)$, predominando o esquema ciclosporina (CSA), azatioprina (AZA) e prednisona (PRED) (tabela 4). A incidência de rejeição aguda foi de $35 \%$ no grupo HCV positivo e $25 \%$ no
HVC negativo ( $\mathrm{p}=0,26)$ e as principais complicações observadas nos dois grupos foram: rejeição aguda e infecções bacterianas. Não houve diferença estatisticamente significante na incidência de diabete melito pós Tx, provavelmente devido ao pequeno número de pacientes analisados. As alterações nas enzimas hepáticas (TGO, TGP e GGT), bem como cirrose, ocorreram apenas em três pacientes HCV positivos (tabela 5).

Tabela 1. Características demográficas dos pacientes

\begin{tabular}{lccc}
\hline & HCV+ & HCV- & Valor $\mathbf{p}$ \\
\hline Idade (receptor) & $37,3 \pm 17,3$ & $32,8 \pm 12,4$ & 0,09 \\
Idade(doador) & $32,0 \pm 10,3$ & $33,8 \pm 12,5$ & 0,4 \\
Sexo (M,F) & $31 / 10$ & $27 / 13$ & 0,31 \\
Cor receptor(B,M,P) & $28 / 6 / 6$ & $38 / 1 / 1$ & 0,08 \\
Tipo doador(DVR,DVNR,DF) & $17 / 1 / 22$ & $23 / 2 / 2015$ & 0,11 \\
HLA (HD,HI,I,NT) & $3 / 8 / 0 / 29$ & $4 / 16 / 2 / 18$ & 0,05 \\
Tempo de Tx(anos) & $12,3 \pm 4,5$ & $12,5 \pm 2,9$ & 0,49 \\
Depuração creat atual (ml/min) & $47,3 \pm 24,9$ & $54,9 \pm 27,2$ & 0,48 \\
Perda do enxerto & $28(70 \%)$ & $34(60 \%)$ & 0,48 \\
Óbito & $14(35 \%)$ & $13(32,5 \%)$ & 0,22 \\
\hline
\end{tabular}

$M=$ masculino, $F=$ feminino, $B=$ branco, $M=$ mulato, $P=$ preto $D V R=$ doador vivo relacionado, $D V N R=$ doador vivo não relacionado, $D F=$ doador falecido, $H D=$ haplodiferente, HI haploidêntico, I = idêntico, $N T=$ não tipado.

Tabela 2. Tempo e causa da perda do enxerto

\begin{tabular}{clll}
\hline & HCV & HCV- & Valor p \\
\hline Tempo até perda (meses) & $36,1 \pm 3,1$ & $47,7 \pm 40,6$ & 0,67 \\
Causa da perda - óbito & $13(32,5 \%)$ & $13(32,5 \%)$ & 1,0 \\
- NCE & $6(15,02)$ & $12(30,0 \%)$ & 0,03 \\
- outras & $21(52,5 \%)$ & $15(37,5 \%)$ & 0,04 \\
\hline
\end{tabular}

NCE = nefropatia crônica do enxerto

Tabela 3. Tempo até óbito e causas do óbito

\begin{tabular}{clll}
\hline & HCV & HCV- & Valor $\mathbf{~ p}$ \\
\hline Tempo até óbito (meses) & $18,0 \pm 30,2$ & $49,8 \pm 58,7$ & 0,03 \\
$\begin{array}{c}\text { Causa/óbito - infecções } \\
\text { bacterianas }\end{array}$ & 5 & 4 & 0,63 \\
$-\begin{array}{c}\text { cancer de pulmão } \\
\text { - hemorragia } \\
\text { pulmonar } \\
\text { - hemorragia } \\
\text { retroperitonial }\end{array}$ & 1 & 0 & - \\
- linfoma & 0 & 1 & - \\
- outras & 1 & 0 & - \\
- indeterminada & 1 & 0 & - \\
\hline
\end{tabular}


Tabela 4. Imunossupressão dos pacientes

\begin{tabular}{lccc}
\hline Tipo & HCV+ & HCV- & Valor p \\
\hline CSA+AZA+PRED & $29(72,5 \%)$ & $28(70,0 \%)$ & 0,87 \\
CSA+AZA+PRED+OKT3 & $5(12,5 \%)$ & $7(17,5)$ & 0,4 \\
CSA+AZA+PRED+BAS & $1(2,5 \%)$ & $1(2,5 \%)$ & 1,0 \\
CSA+MMF+PRED & $3(7,5 \%)$ & $2(5,0 \%)$ & 0,85 \\
CSA+SRL+PRED & $1(2,5 \%)$ & $1(2,5 \%)$ & 1,0 \\
TCA+MMF+PRED & $1(2,5 \%)$ & $1(2,5 \%)$ & 1,0 \\
\hline
\end{tabular}

CSA = ciclosporina neoral, $A Z A=$ azatioprina, $O K T 3=$ orthoclone,

$B A S=$ basiliximab, $S R L=$ sirolimo, $T A C=$ tacrolimo, $P R E D=$ prednisona

Tabela 5. Complicações dos transplantes

\begin{tabular}{lccc}
\hline Tipo & HCV & HCV- & Valor P \\
\hline Rejeição aguda & $14(35,0 \%)$ & $10(25,0 \%)$ & 0,36 \\
Infecções bacterianas & $9(22,5 \%)$ & $10(25,0 \%)$ & 0,79 \\
Necrose tubular aguda & $9(22,5 \%)$ & $6(15,0 \%)$ & 0,39 \\
Nefrotoxicidade por CSA & $6(15,0 \%)$ & $6(15,0 \%)$ & 1 \\
Citomegalovirose & $3(7,5 \%)$ & $1(2,5 \%)$ & 0,61 \\
Alterações dos enzimas & $2(5,0 \%)$ & 0 & 0,47 \\
Diabete melito pós Tx & $5(12,5 \%)$ & $1(2,5 \%)$ & 0,2 \\
Cirrose Hepática & $1(2,5)$ & 0 & 0 \\
\hline
\end{tabular}

\section{DISCUSSÃO}

A história natural de hepatite por HCV em pacientes submetidos a Tx renal ainda não está bem estabelecida. Alguns autores acreditam que a imunossupressão facilita a replicação viral e agrava a doença hepática. ${ }^{12,13}$ Entretanto, um aumento na carga viral pode não se associar a um aumento no risco da doença hepática após o Tx. ${ }^{6,14,15}$
De acordo com a literatura, o mau resultado do Tx em pacientes com HCV decorre da elevada mortalidade e morbidade determinadas pela doença hepática e infecção bacteriana. ${ }^{6,12,13,16}$ Insuficiência hepática constitui a principal causa de morte a longo prazo em 8 a 28\% dos casos., ${ }^{1,5}$ No nosso estudo, a principal causa de óbito nos pacientes HCV positivos e negativos foi decorrente à infecção bacteriana, de incidência semelhante em ambos os grupos. Cirrose hepática e alterações nas enzimas hepáticas ocorreram em apenas três pacientes HCV positivos, porém, não foram responsáveis pelo óbito dos pacientes, contrariando o observado na literatura., ${ }^{9,17}$

A sobrevida dos pacientes em ambos os grupos estudados foi semelhante, porém, o óbito foi mais precoce nos pacientes HCV positivos. Não houve diferença significativa na sobrevida do enxerto entre pacientes HCV positivos e negativos, o que está de acordo com algumas publicações encontradas na literatura, ${ }^{18,19}$ contrariando outros estudos.,13

A incidência de rejeição aguda em pacientes infectados por HCV ainda é controversa. Algumas publicações têm demonstrado elevada freqüência entre os pacientes $\mathrm{HCV}$ positivos quando comparados com os HCV negativos. ${ }^{12,20,21}$ Entretanto, não foi encontrada diferença significativa na incidência da rejeição aguda nos grupos estudados no presente estudo.

A prevalência do diabete melito foi pequena, não ocorrendo diferença estatisticamente significante entre grupos, ao contrário do que se observa na literatura. ${ }^{22,23}$ Provavelmente, isso foi devido ao predomínio quase absoluto da imunossupressão com CSA e ao pequeno número de pacientes analisados. Apenas dois pacientes receberam tacrolimo, que é uma droga potencialmente diabetogênica, principalmente em pacientes HCV positivos.

\section{CONCLUSÃO}

Este estudo mostrou que a infecção por vírus $C$ não teve impacto na sobrevida dos pacientes, do enxerto, bem como no aumento da incidência da infecção bacteriana, rejeição aguda e insuficiência hepática. São necessários estudos prospectivos com número maior de pacientes, incluindo carga viral e análise do genótipo para melhor definir o impacto do HCV no transplante renal em longo prazo. A observação dos pacientes deverá continuar, para que se observe o resultado em longo prazo.

\section{ABSTRACT}

Purpose: The authors assessed the impact on the kidney transplantation associated to the $\mathrm{C}$ virus chronic hepatitis (HCV) infection, analyzing the complications, patients' and graft survival. Methods: Retrospective study of 40 kidney transplant recipients bearers of HCV infection and 40 kidney transplant recipients no-bearers of HCV infection along the same post transplantation period. Results: The mean post transplantation time was $12.3 \pm 4.5$ years of patients bearers of HCV infection, and $12.5 \pm 2.9$ years of patients no-bearers of HCV infection $(\mathrm{P}=0.49)$. There was no statistical difference as to the age and gender of recipients or donors. The present renal function in patients bearers of $\mathrm{HCV}$ infection was $47.3 \pm 24.9 \mathrm{ml} / \mathrm{min}$, and $54.9 \pm 27.2 \mathrm{ml} / \mathrm{min}$ in the group of no bearers $(\mathrm{p}=0.48)$. The incidence of the graft and patient survival was similar in both groups. The main cause of death in both groups was bacterial infection (10\% in HCV patients and $12.5 \%$ in the group of no bearers, $\mathrm{p}=0.63$ ). The most common complication between both groups was acute allograft rejection and bacterial infection, and the incidence of diabetes mellitus showed no statistical difference. Abnormal levels of liver enzymes and cirrhosis were only observed in patients bearers of HCV infection. Conclusion: HCV infection did not show to have impact on the patient and graft survival and on complications in the post transplantation.

Keywords: HCV, Kidney Transplantation, Chronic Kidney Failure 
918 Euler Pace Lasmar, Marcus Faria Lasmar, Leonardo Faria Lasmar, Adalberto Fernandes Nogueira, Marina Ribeiro de Oliveira Santos, Luiz Flávio Couto Giordano, Heloisa Reniers Vianna, Juliene Borges Fujji

\section{REFERÊNCIAS}

1. Fabrizi F, Poodad F, Martin P. Hepatites C infection and the patient with end stage renal disease. Hepatology. 2002;36(1):3

2. Vosnides GG. Hepatites C in renal transplantation. Kidney Int. 1997;52 (3):843

3. Bloom RD, Sayer G, Fak et al: Outcome of hepatitis C virus-infected kidney transplant candidates who remain on the waiting list. Am I Transplant. 2005:5:139

4. Sezer S, Ozdemir FN, Akcay A et al: Renal transplantation offers a better survival in HCV infected ESRD patients. Clin Transplant. 2004;18:G19

5. Pereira BJ: Hepatitis C infection and post-transplantation liver disease.Nephrol Dial Transpl. 1995;10 (suppl1):58

6. Pereira BJ, Wright TL, Schmid CH et al:The impact of pre transplantation hepatitis C infection on the outcome of renal transplantation.Transplantation. 1995;60:799

7. Gentil MA, Rocha JL, Rodrigues-Algarra G et al: Impaired kidney transplant survival in patients with antibodies to hepatitis C virus. Nephrol Dial Transplant 1999; $14: 2453$

8. Stempel CA, Lake J, Kuo G et al: Hepatitis C virus infection and renal transplantation. Transplantation. 1993;55:273

9. Sahin GM, Sahin S, Kantarcig and Ergin H. Impact of hepatitis C virus infection on patient and graft survival in kidney transplantation.Transplant Proc. 2006;38:499-501

10. Pedroso S, Martines L, Fonseca I et al.Impact of hepatits $\mathrm{C}$ virus on renal transplantation:association with poor survival.Transplant Proc. 2006;38:1890-4

11. Corrêa Jr, Rocha FD, Gonçalves LF, Manfro RC. Long term effect of hepatitis B and $\mathrm{C}$ virus on the survival of kidney transplant patients. Rev Assoc Med Bras. 2003;49:389-94

12. Morales JM, Campistol JM: Transplantation in the patient with hepatitis C.J Am Soc Nephrol. 2000;11:1343
13. Logendre $\mathrm{CH}$, Garrigue V, Le Bihan $\mathrm{C}$ et al: Harmful long-term impact of hepatitis C virus infection in Kidney transplant recipients.Transplantation. 1998;65:667

14. Roth D, Zucker K, Cirocco R et al: The impact of hepatitis C virus infection on renal allograft recipients. Kidney Int. 1994;45:238

15. Kokado Y, Takahara S, Ichimaru Netal: Clinical outcome of HCV infection after renal transplantation. Transplant Proc. 2000;32:1940

16. Knoll GA, Tankersle MR, Lec LY et al: The impact of renal transplantation on survival in hepatitis C positive end-stage renal disease patients.Am J Kidney Dis. 1997;29:608

17. Morales JM, Domingues-Gil B,Sanz-Gajardo D et al: The influence of hepatitis B and hepatitis $C$ vírus infection in the recipient on late allograft failure.Nephrol Dial Transplant. 2004;19:572

18. Pereira BJ. Effects of hepatitis $\mathrm{C}$ infection and renal transplantation on survival in end stage renal disease.The New England Organ Bank Hepatitis C Study Group. Kidney Int. 1998;53:1374

19. Lee WC, Shu KH, Cheng $\mathrm{CH}$ et al:Long-term impact of hepatitis $\mathrm{B}, \mathrm{C}$ virus infection on renal transplantation. Am J Nephrol. 2001;21:300

20. Morales JM:Renal transplantation in patients positive for hepatitis B ou C. Transplant Proc. 1998;30:2064

21. Corell A, Morales JM, Mandrono A et al: Immunosuppression induced by hepatitis C virus infection reduces acute renal transplant rejection. Lancet. 1995;346:1497

22. Manga G, Sahin S, Kantarci e tal: Impact of hepatitis C virus infection on patient and graft survival in kidney transplantation. Transp Proc. 2006;38:499-501

23. Stehman-Breenco, Emerson S, Gretch D et al. Rise of death among chronic dialysis patients infected with hepatitis C virus. Am J Kidrey Dis. 1998;32:629. 